\title{
L'instrumentation dans les stations d'épuration urbaines
}

\author{
Hervé Wacheux \\ Anjou Recherche, Chemin de la Digue, BP 76, 78600 Maisons-Laffitte
}

Tél. : 16 (1) 39623456

\section{Introduction}

La présence de nitrates dans les eaux destinées à la potabilisation, l'eutrophisation par les phosphates de nombreuses ressources en eau ont conduit les collectivités et les agences de bassin à promouvoir la construction de stations d'épuration éliminant l'azote et, dans quelques cas, du phosphore. Les niveaux de rejets NGL $1(20 \mathrm{mg} / 1 \mathrm{de} \mathrm{N})$ voir NGL 2 (10 mg/l de N) sont maintenant demandés en de nombreux endroits.

Une seconde tendance commence seulement à s'esquisser. Il s'agit de l'élimination du phosphore. En effet, environ la moitié des rejets de phosphore dans le milieu naturel provient des agglomérations.

Les directives européennes du 21 mai 1991 sur les eaux usées sont en cours de transcription dans la législation française. Les principaux apports concernent la distinction entre des zones normales oủ le niveau de rejet est du même ordre que le niveau e français, et des zones sensibles pour lesquelles il est demandé l'élimination de l'azote global et du phosphore. En outre, elles autorisent des rejets de moins bonne qualité si des rendements d'épuration sont atteints.

Un second point extrêmement nouveau concerne la tenue des niveaux de rejet. En effet, dans un domaine d'applicabilité fixé, un nombre minimum d'analyses par an doit être effectué sur les rejets et un nombre maximum d'analyses non conformes est toléré. Ce nombre de défaillances est établi sur la base d'une répartition statistique garantissant à tout moment (avec une probabilité de $95 \%$ ) d'être en deçà des concentrations limites autorisées.

L'élimination de l'azote peut s'effectuer par voie biologique dans des procédés classiques comme les stations à aération prolongée ou dans des procédés sophistiqués comme le Biocarbone ou le Biostyr développés par OTV qui remplacent le bassin de boues activées et clarificateur, qui économisent l'espace et s'intègrent facilement dans l'espace urbain.
L'élimination du phosphore est souvent complétée ou remplacée par la voie physico-chimique (précipitation des phosphates par le chlorure ferrique) qui est simple à mettre en œuvre mais coûteuse en exploitation. Par conséquent, il est nécessaire de doser les réactifs d'une manière optimale.

L'élimination de l'azote et du phosphore induit une multiplication des réacteurs et des actions à mener simultanément de sorte que si l'on n'y prenait garde, on assisterait à une fragilisation globale du procédé. Pour garantir une bonne fiabilité et une optimisation de ces nouvelles stations d'épuration, il est nécessaire de prévoir dans l'ordre : une bonne conception, un bon dimensionnement, une mise en œuvre correcte et des moyens d'exploitation adéquats. Ceux-ci consistent pour les grosses installations en un ensemble d'automates, de superviseurs et de télétransmission s'appuyant à la base sur des capteurs en nombre suffisant pour permettre une bonne connaissance du procédé.

Il est nécessaire de procéder à des boucles de recirculation car le produit brut entrant dans une station d'épuration est un flux continu et variable dans le temps tant en quantité qu'en qualité, alors que le produit fini qui sort de la station doit être une eau claire, pratiquement exempte de matières organiques, d'azote et de phosphore à des niveaux parfois inférieurs à ceux rencontrés dans l'eau potable. De plus, la qualité de l'eau en sortie doit être peu variable de façon à rester en moyenne journalière audessous des valeurs limites autorisées.

La pratique actuelle de conduite des stations d'épuration à base d'horloges journalières ou hebdomadaires fait largement appel aux connaissances de l'opérateur sur les chroniques d'apport et sur la réaction de la station à une modification des conditions de fonctionnement. Il en résulte un mode de conduite assez figé (par crainte de dérèglement) et à la progression hasardeuse. 


\section{Niveau actuel d'équipement des stations d'épuration}

En dehors du débitmètre de sortie, souvent requis par les agences de bassin et à des fins d'établissement des primes d'épuration, l'instrumentation des stations d'épuration est quasi-inexistante. Pourquoi ?

- Tout d'abord, par le mécanisme de l'attribution des marchés publics au moins disant qui conduit les soumissionnaires à éliminer tout ce qui est superflu ou non indispensable. Evventuellement, ils proposeront en option ce qui est commercialement attractif, c'est-à-dire le système de contrôle-commande avec ses écrans ou synoptiques riches en couleur et de haute technologie. Mais c'est insuffisant pour la conduite qui nécessite avant tout des capteurs d'information sur le procédé.

- En second lieu, les acteurs dans ce domaine se méfient de l'instrumentation. Des expériences malheureuses dans le

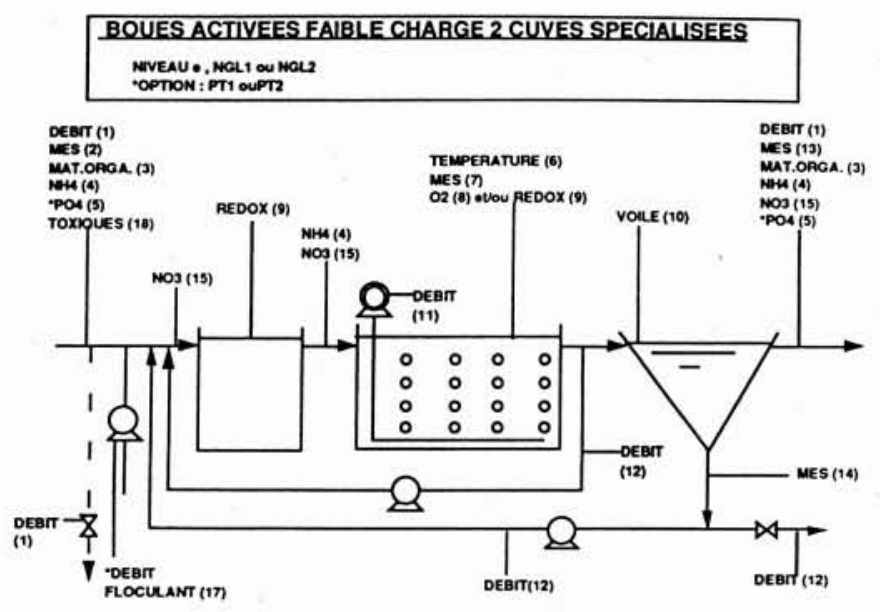

1.

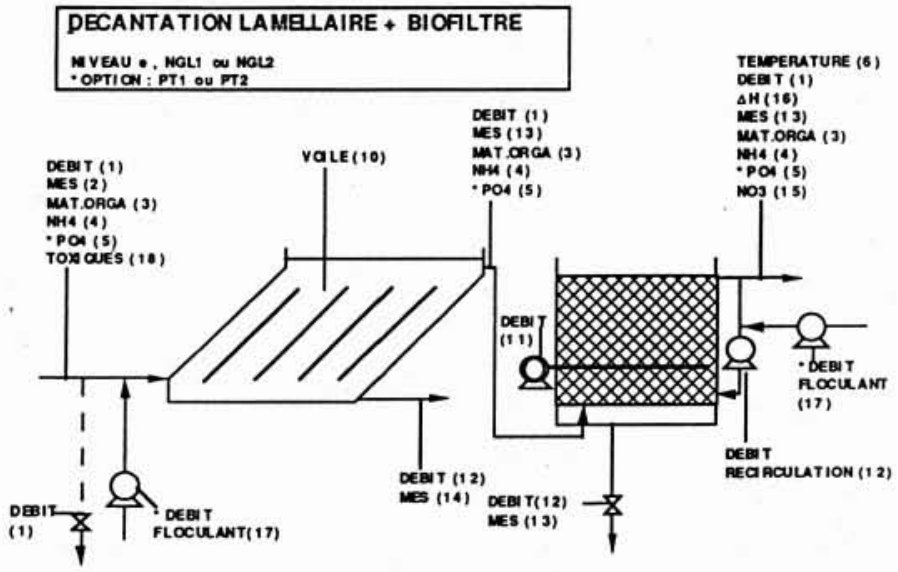

2. passé ont convaincu beaucoup d'exploitants que les capteurs dans ce domaine étaient peu fiables, peu précis et demandaient beaucoup de maintenance pour des résultats aléatoires.

- De plus, l'ensemble de l'instrumentation n'est pas génératrice d'économie de fonctionnement. Seul le capteur d'oxygène dissous, par les économies d'énergie qu'il permet au niveau de l'aération a connu un assez grand succès. Mais il n'équipe pas encore, loin sans faut, tous les bassins d'aération. La conduite des stations est déjà automatique, essentiellement à base d'horloges, et il n'y a donc pas de possibilité de réduire le personnel qui est déjà extrêmement réduit. L'apport de l'instrumentation se situe au niveau de la fiabilité et de l'optimisation du traitement et ne se traduit par aucun retour financier pour la collectivité ou le gestionnaire.

- Enfin la mise en place d'un ou deux capteurs a un effet limité, car il en faut davantage pour pouvoir réguler, optimiser et diagnostiquer l'ensemble de la station.

Pourtant, les arguments en faveur de l'instrumentation ne manquent pas :

- Les technologies ont évolué et les capteurs qui pouvaient paraître superflus deviennent de plus en plus indispensables dans des installations qui se compliquent et aux marges de sécurité étroites.

- La fiabilité des stations qui était un critère flou dans le passé commence à se préciser. Dans les directives européennes, 6 à $10 \%$ d'analyses non conformes sont autorisées, mais pour assurer complètement le respect d'un niveau de rejet, il n'existe pas d'autre alternative que l'instrumentation en continu.

- L'auto-contrôle réalisé de façon classique par prélèvements et analyses en laboratoire a un temps de réponse minimum de 48 heures, ce qui est trop long pour pouvoir être utilisé pour une correction de la conduite. De plus, il est généralement pratiqué sur une minorité de jours de l'année et peut donc passer à côté de problèmes de peu de durée.

- Plus le niveau de rejet est élevé, plus le milieu récepteur se reconstitue et retrouve une fragilité aux pollutions accidentelles. Par suite, tout incident au niveau de la station prend des proportions inquiétantes et visibles. La sauvegarde de l'état du milieu naturel passe désormais par un lissage des rejets plutôt que par l'abaissement du niveau moyen des flux polluants.

\section{Définition de l'information à acquérir}

Pour définir quelle est l'instrumentation souhaitable dans une station d'épuration en dehors du traitement des boues, on peut envisager à titre d'exemple les deux schémas de filière qui sont présentés dans les figures 1 et 2 :

- le premier concerne une station d'épuration à boues activées faible charge ;

- le second concerne une décantation lamellaire suivie d'un biofiltre. 
Dans une station d'épuration à boues activées à deux cuves, éliminant l'azote, il est nécessaire de gérer le débit admis, l'aération, la recirculation des boues et des liqueurs mixtes, ainsi que l'extraction des boues. Pour cela, les modes de conduite traditionnels à base d'horloges ne suffisent plus, il faut mettre en place des régulations à partir d'informations issues de capteurs en ligne.

Si de surcroit, elle effectue l'élimination biologique du phosphore, il convient de régler le temps de séjour dans la cuve anaérobie et l'aération dans les bassins pour ne pas relarguer les phosphates.

Il existe trois natures d'information

- La première concerne les quantités ou volumes. En effet, il est essentiel de connaître les débits entrants, déversés par le by-pass, recirculés, extraits par les boues ainsi que les débits épurés.

- La seconde concerne la qualité ou caractérisation du produit reçu (l'influent) et sa modification le long de la filière jusqu'au rejet du produit épuré (l'effluent). Cette qualité peut être estimée de diverses manières: MES (Matières en Suspension), DCO (Demande Chimique en Oxygène), $\mathrm{DBO}_{5}$ (Demande Biologique en Oxygène), $\mathrm{NH}_{4}$ (ammoniaque), NTK (azote total Kjeldal), $\mathrm{NO}_{3}$ (nitrate), $\mathrm{P}_{\text {tot }}$ (phosphore total), $\mathrm{PO}_{4}$ (orthophosphates)... etc.

- La troisième concerne des paramètres utilisés strictement pour la conduite: oxygène dissous, $\mathrm{pH}$, redox, température, respiration de boue, décantabilité...

La mesure de débit est indispensable pour établir les flux traités et les rendements au long de la filière, elle permet la gestion des recirculations et extractions de boues ainsi que le dosage des réactifs.

La MES est la mesure de qualité la plus simple et la moins coûteuse à réaliser en ligne via la turbidité. Aussi trouve-t-on cette mesure en entrée, dans le bassin des boues activées, sur le retour et l'extraction de boues, dans les décanteurs primaires et secondaires, en sortie sur l'effluent. Des études réalisées à Arras et à St Omer ont montré qu'elle était souvent aussi un bon indicateur de la DCO.

La DCO, qui se prête mal à une mesure en continu en ligne a été remplacée par le COT au niveau de nombreux rejets industriels. Cette mesure est encore peu répandue dans les stations d'épuration en raison du coût des COT mètres et du maintien de la DCO comme indicateur de référence. Cette mesure serait pourtant très utile pour établir le bilan entrée-sortie des charges et le rendement. De plus, elle mettrait en évidence les pointes et surcharges de la station.

$\mathrm{La} \mathrm{DBO}_{5}$ est un indicateur d'oxydabilité biologique dans le milieu naturel mais présente déjà des difficultés de reproductibilité en laboratoire et se prête mal vu le temps qu'elle demande à une mesure en ligne. Comme la DCO, elle permettrait l'appréciation d'un rendement d'épuration, des pointes et surcharges.

L'azote $\mathrm{N}$ sous ses deux formes principales $\mathrm{NH}_{4}$ et $\mathrm{NO}_{3}$ est d'un grand intérêt dans les stations d'épuration qui éliminent l'azote, à la fois pour produire des bilans entréesortie et pour contrôler chaque phase de l'épuration le long de la filière.
L'azote global, retenu par les directives européennes, peut être mesuré en ligne mais compte tenu des coûts et de la complexité de ces analyseurs, il est préférable de mesurer séparément les différentes espèces azotées.

Le phosphore total est également un paramètre retenu par les directives européennes mais pour la mesure en ligne il lui est préféré les orthophosphates $\left(\mathrm{PO}_{4} \ldots\right)$. Cette mesure peut intervenir en entrée-sortie pour le contrôle des rejets et l'établissement de rendements, mais aussi dans la filière notamment pour la régulation de réactif dans le cas d'une déphosphatation physico-chimique.

Enfin, parmi les paramètres utilisés strictement pour la conduite, on trouve :

- la perte de charge à travers un biofiltre pour estimer son colmatage ainsi que l'efficacité du lavage ;

- l'oxygène dissous dans les bassins d'aération pour réguler l'aération et maintenir l'oxydant $\mathrm{O}_{2}$ à un niveau suffisant ;

- le redox dans les bassins de boue activée en alternance de phase pour réguler les phases de marche et d'arrêt des aérateurs ;

- la température dans les bassins biologiques pour apprécier son incidence sur les cinétiques en particulier sur la nitrification ;

- la toxicité en entrée pour gérer un stockage en bassin et protéger la biomasse épuratrice ;

- la décantabilité des boues pour le contrôle du clarificateur.

\section{Passage en revue des capteurs et technologies exis- tantes}

Sur le plan technologique, on peut classer les différentes informations précédentes en quatre groupes:

- Débits, niveau, pression : ce sont des mesures physiques non spécifiques à l'eau et très répandues dans l'industrie. - Conductivité, pH, redox, oxygène dissous : ce sont des mesures électrochimiques moins répandues que les précédentes et pour lesquelles la spécificité à l'eau usée est partielle.

- Turbidité, MES, capteurs à ultra-sons: ce domaine relève essentiellement de l'optique, la spécificité à l'eau usée est assez forte.

- DCO, $\mathrm{DBO}_{5}, \mathrm{COT}, \mathrm{NH}_{4}, \mathrm{NO}_{3}, \mathrm{PO}_{4}, \ldots$ : : il s'agit d'automates d'analyse dérivés des automates pour laboratoire et qui ont ensuite été utilisés pour le contrôle de qualité des rejets industriels et des eaux destinées à la potabilisation.

\section{Débit, niveau, pression}

Pour les débits dans les conduites en charge, la technique la plus employée est le débitmètre électromagnétique à champ commuté ou alternatif. Celui-ci présente des difficultés lorsque le fluide est suceptible d'isoler les électrodes (graisses, croûte organique, ...). Il est utilisé sur les circuits de retour ou d'extration des boues, sa précision est de l'ordre du pourcent. 


\section{H. WACHEUX}

Les nouveaux capteurs de débit massique, à effet Coriolis ne sont pas encore utilisés dans les stations. Ils présentent pourtant des caractéristiques recherchées dans le domaine des eaux usées comme l'insensibilité aux MES.

Pour la mesure des débits à surface libre : deux principes sont utilisés :

- soit utiliser un obstacle qui rend le débit fonction uniquement de la hauteur d'eau en amont, comme les déversoirs, venturis, parshalls,... etc. ;

- soit mesurer la hauteur, en déduire la section mouillée, mesurer une vitesse, en déduire la vitesse moyenne et enfin le débit. Pour la mesure de la vitesse, on utilise des paires de sondes à ultra-sons, des moulinets électromagnétiques ou à effet Doppler.

La mesure par venturi + hauteur d'eau est la plus simple à maintenir et à calibrer.

Pour la mesure des niveaux, on utilise des sondes à ultra-sons placées au-dessus de la surface, ou des capteurs de pression (piezo-résistifs, capacitifs ou bulle à bulle) dans le fond du chenal. Les premiers craignent les mousses et débris flottants, les échos parasites ... et les seconds sont plus exigeants en maintenance et calibration et sont moins précis dans les conditions habituelles d'emploi.

\section{Conductivité, $p H$, redox, oxygène dissous}

Conductivité et $\mathrm{pH}$ sont peu employés dans les stations d'épurations urbaines. Ils sont sensibles à une salissure des électrodes et nécessitent une maintenance active.

Le redox est de plus en plus utilisé dans les petites stations d'épuration du groupe Compagnie Générale des Eaux pour la régulation de l'aération. Ce capteur non spécifique rend bien compte de l'état global oxydé ou réduit du milieu. Les électrodes sont sensibles à une salissure et même à une contamination notamment par $\mathrm{H}_{2} \mathrm{~S}$. L'emploi de sondes combinées est préférable. La maintenance est essentielle pour l'utilisation en régulation.

L'oxygène dissous est le capteur le plus populaire après le débi-mètre en sortie. Ils sont sensibles à la formation d'un biofilm sur la membrane, ainsi qu'au vieillissement et à la contamination du capteur. Il est préférable d'utiliser des sondes à profil ne favorisant pas l'accrochage ou les dépôts, à membrane et anode de grande taille, à tête jetable et à compensation de température soignée. Des essais effectués à Anjou Recherche ont montré des temps de réponse de 1 à 40 minutes, des écarts de 10 à $20 \%$ après calibrage et des résistances à l'encrassement très variables.

Toutes ces mesures sont ponctuelles alors que l'objectif visé est la moyenne, surtout dans le cas d'une installation en bassin. Le choix de l'implantation du capteur et/ou d'une multiplication des points de mesure sont des facteurs essentiels.

\section{Turbidité, MES}

Le principe le plus employé est la diffusion d'un faisceau lumineux par les particules. Les gammes étant variées (depuis quelques $\mathrm{mg} / \mathrm{l}$ jusqu'à $100 \mathrm{~g} / \mathrm{l}$ ), il est fait appel à la néphélométrie pour les eaux claires, l'opacimétrie pour les boues activées et la rétrodiffusion dans les gammes les plus élevées. Les essais effectués par Anjou Recherche en entrée de station, en bassin d'aération et en sortie de station, ont amené aux conclusions suivantes :

L'auto-nettoyage est souhaitable ou indispensable selon les cas. La mesure in-situ est préférable pour éviter les pompes et les conduites qui risquent de biaiser l'échantillon avant la mesure.

Pour pallier l'encrassement qui reste la première cause de disfonctionnement des turbidimètres en station d'épuration, plusieurs techniques ont été mises en œuvre :

- mesure sur un jet d'eau en chute libre ;

- nettoyage par diffusion à la surface de l'eau ;

- nettoyage aux ultra-sons ;

- nettoyage par râcleur ou piston ;

- nettoyage par jet d'eau ou d'air ;

- compensation électronique.

Le plus efficace à long terme et le plus facile à mettre en œuvre nous semble être le nettoyage par râclage mécanique.

Pour faciliter la maintenance, l'étalonnage et l'installation, un capteur immergé de faibles dimensions est souhaitable, tandis que pour prendre en compte la coloration éventuelle il est préférable d'effectuer une mesure suivant deux directions.

Il est préférable d'effectuer des mesures de diffusion vers l'avant. Or, compte-tenu des valeurs importantes d'absorption dans les bassins ou pis dans les épaississeurs, ceci n'est guère possible avec des tailles de cellule de mesure raisonables, aussi la rétro-diffusion est elle alors employée. D'autres alternatives apparaissent actuellement : la mesure de l'absorbance d'un faisceau d'ultra-sons, d'un faisceau de rayons gamma, ou d'un faisceau d'ondes hyperfréquence.

Il existe des capteurs de MES un peu particuliers, ce sont les capteurs de voile de boue en position fixe ou mobile pour les décanteurs, clarificateurs et épaississeurs.

Dans le premier cas, il s'agit de capteurs de MES dentiques à ceux pour les bassins de boues activées mais munis de seuils. Dans le second cas, il s'agit de capteurs de MES suspendus à un câble enroulé sur un treuil motorisé. $\mathrm{Ce}$ dernier est asservi à une valeur de MES représentant le voile de boue. Le capteur de MES peut être selon le cas un barreau vibrant, un couple émetteur-récepteur d'ultrasons ou un turbidimètre optique.

Enfin, dans cette catégorie, nous terminerons par les capteurs d'indice de décantabilité des boues. Il s'agit d'une automatisation d'un test de décantation de type indice de Molhman ou SSVI. Ces capteurs sont encore à un stade expérimental mais ils offrent des perspectives intéressantes pour le contrôle du foisonnement des boues.

Les capteurs de COT, $\mathrm{DBO}, \mathrm{NH}_{4}, \mathrm{NO}_{3}, \mathrm{PO}_{4}$, toxicité,...

Les COTmètres effectuent une oxydation à chaud dans un four, ou à froid par UV ou ozone. Une évaluation des principaux COTmètres a été effectuée par l'Exéra.

Le système d'échantillonnage et de conditionnement de l'échantillon est encore source de nombreux problèmes et 
représente un coût élevé à l'achat.

$\mathrm{La} \mathrm{DBO}_{5}$ est encore bien moins propice à une mesure en continu. La respirométrie qui est utilisée à Anjou Recherche depuis une dizaine d'années a été employée pour une estimation de la DBO sur la base d'une respiration pendant une à trois minutes. Il ne s'agit là que d'un estimateur et non de la procédure normalisée. Dans le cas de rejets urbains, la respiration instantanée, de même que $\mathrm{NH}_{3}$ ou la turbidité, est assez bien corrélée avec la DCO et la DBO.

Les ions $\mathrm{NH}_{4}^{+}, \mathrm{NO}_{3}^{-}, \mathrm{PO}_{4}^{--}$sont réputés être entièrement en solution dans les conditions ordinaires. Le dosage de l'ammoniaque peut s'effectuer par colorimétrie (Nessler, Bertholst, Salicilate) ou par électrode spécifique. La mesure de $\mathrm{NH}_{3}$ par électrode est la plus employée dans les analyseurs automatiques avec des électrodes gaz- $\mathrm{NH}_{3}$ ou ion $\mathrm{NH}_{4}^{+}$. Le dosage des nitrates en laboratoire est souvent réalisé par réduction des nitrates en nitrites et dosage de ces derniers par diazotation.

Les autres méthodes sont les électrodes à ion spécifique $\mathrm{NO}_{3}$ et l'absorption dans l'UV.

Chaque méthode présente des interférents qui lui sont en partie spécifique : la coloration affecte la colorimétrie et l'UV, les ions forts affectent les électrodes à ion spécifique. Une amélioration du procédé de mesure consiste à effectuer des ajouts dosés comme le fait Hydro Environnement.

Le dosage des orthophosphates en laboratoire peut s'effectuer par HPLC, RMN ou isotachoporèse mais plus classiquement par colorimétrie à l'acide vanadomolybdique avec réduction éventuelle à l'acide ascorbique, au chlorure d'étain ou l'acide aminonaphtolsulfonique.

Parmi les autres capteurs, il en existe aussi qui pourraient être utilisés dans certains cas particuliers. Il s'agit de capteurs d'hydrocarbures ou huiles flottantes, basés sur un principe d'absorption d'ondes hyperfréquence, ou encore d'analyseurs de détergents ioniques basés sur des principes d'électrochimie.

Quel pourrait être un équipement asséz complet sur une station d'épuration. Prenons le cas de la station d'épuration de Meyzieu ( $35000 \mathrm{eq} / \mathrm{hbts}$ ) dont le système de gestion a été réalisé par OTV en association avec la COURLY.

Le procédé consiste en une décantation primaire par décanteurs lamellaires suivie d'une épuration par biofiltre Biocarbone.

Seize capteurs permettent de suivre le procédé :

- eau brute : débit, MES ;

- eau décantée primaire : débit, MES, absorption UV, absorption visible, température ;

- eau épurée : débit, absorption UV, absorption visible ;

— air : débit ;

— boues primaires : débit, MES ;

— boues épaissies : débit, MES.

Prenons un autre exemple, celui de la station d'épuration de St Omer (110000 eq/habts) dont le schéma est joint sur la figure 3 .

Il s'agit d'une boue activée faible charge à deux cuves spécialisées anoxie et aérobie. Elle comprend 17 capteurs répartis le long du procédé :
- eau brute : débit, $\mathrm{Nh}_{4}, \mathrm{MES}, \mathrm{pH}$;

- by-pass : débit ;

— bassin d'aération : température, oxygène, MES ;

- air process : débit ;

- clarificateur : voile de boue, débit des boues recirculées, débit et MES des boues extraites; - eau épurée : débit, $\mathrm{NH}_{4}, \mathrm{MES}$.

STEP ST OMER : CAPTEURS

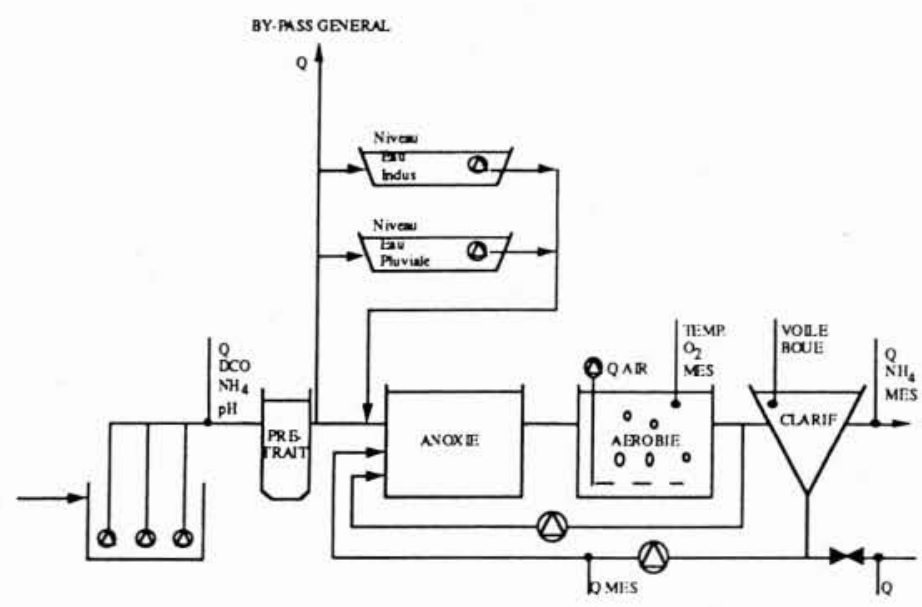

STEP ST OMER : LES AUTOMATISMES

\begin{tabular}{|c|c|c|c|c|}
\hline & CAPTEURS & ACTIONNEURS & CONSIGNES & REGULATION \\
\hline AERATION & sonde $\mathrm{O}_{2}$ & $\begin{array}{c}\text { vitesse } \\
\text { compresseur }\end{array}$ & $\begin{array}{l}24 \mathrm{O} / \mathrm{J} \\
7 \text { jours }\end{array}$ & P.I.D \\
\hline $\begin{array}{l}\text { EXTRACTION } \\
\text { BOUES }\end{array}$ & $\begin{array}{l}\text { Q boues } \\
\text { MES boues }\end{array}$ & pompe à boues & $\begin{array}{l}\text { I MES bassin } / \mathrm{j} \\
7 \text { jours }\end{array}$ & prog. automate \\
\hline RECIRC. BOUES & ...... & $\begin{array}{l}\text { Pompe de } \\
\text { recire. boues }\end{array}$ & $\begin{array}{c}4 \text { Q recirc } / \mathrm{J} \\
7 \text { jours }\end{array}$ & P.I.D \\
\hline $\begin{array}{l}\text { RECIRC. LIQ } \\
\text { MIXTES }\end{array}$ & ....... & $\begin{array}{l}\text { Pompe recirc. } \\
\text { liqueur mixte }\end{array}$ & $\begin{array}{c}4 \text { rec liq mix/j } \\
7 \text { jours }\end{array}$ & AM sur seuil \\
\hline $\begin{array}{c}\text { STOCKAGE EAU } \\
\text { PLUIE }\end{array}$ & Q ent STEP & $\begin{array}{l}\text { pompe relev. }{ }^{+} \\
\text {vanne murale }\end{array}$ & $\begin{array}{l}1 \text { Qmax pour } \\
\text { les } 7 \text { jours }\end{array}$ & P.I.D sur seuil \\
\hline $\begin{array}{l}\text { STOCKAGE EAU } \\
\text { INDUSTR. }\end{array}$ & pH entrée & $\begin{array}{l}\text { pompe relev. }{ }^{+} \\
\text {vanne murale }\end{array}$ & $\begin{array}{c}1 \mathrm{pHmin}, 1 \mathrm{pH} \\
\max \\
1 \mathrm{Q} \text { réintrod }\end{array}$ & P.I.D sur seuil \\
\hline
\end{tabular}

3.

Le tableau qui est joint montre l'utilisation de ces capteurs en régulation. 


\section{Conclusion}

Ce rapide tour d'horizon sur l'instrumentation dans les stations d'épuration montre que l'offre en capteurs est assez fournie bien que parfois assez lointaine des paramètres ayant une reconnaissance officielle.

Le niveau d'équipement des stations d'épuration françaises en capteurs est très faible et bien inférieur à celui de nos voisins allemands. Le besoin en capteurs pour la conduite et pour le suivi des performances commence seulement à s'exprimer. La nécessité de l'équipement en capteurs ne sera réelle qu'avec l'appui des organismes de contrôle et de la législation.

Pour les petites stations d'épuration, un équipement limité pourrait consister en un débitmètre en sortie, un oxymètre ou redox dans le bassin aéré et un turbidimètre en sortie.

Pour les grosses stations d'épuration éliminant l'azote et le phosphore, une vingtaine ou une trentaine de capteurs sont nécessaires pour suivre le procédé dans sa totalité. Il est souhaitable que des stations d'épuration comme celle de Meyzieu et celle de St Omer fassent école et que leur équipement en capteurs soit considéré comme normal dans les nouvelles stations. 